\title{
Health Facility Preparedness and Response to COVID-19: An Assessment of Employee Satisfaction in a Teaching Hospital in Southern Nigeria
}

\author{
Esohe Olivia Ogboghodo, ", Joy Chinyere Nwaogwugwu ${ }^{1}$, Otaniyenuwa Eloghosa Obarisiagbon ${ }^{1}$, \\ Orezimena Temitope Omo-Ikirodah ${ }^{1}$, Edith Imuwahen Uwugiaren ${ }^{2}$, Obehi Aituaje Akoria ${ }^{3}$, \\ Darlington Ewaen Obaseki ${ }^{4}$, Mokogwu Ndubuisi ${ }^{1}$
}

${ }^{1}$ Department of Community Health, University of Benin Teaching Hospital, Benin City, Nigeria
${ }^{2}$ Department of Nursing Services, University of Benin Teaching Hospital, Benin City, Nigeria
${ }^{3}$ Department of Geriatrics, University of Benin Teaching Hospital, Benin City, Nigeria
${ }^{4}$ Department of Anatomic Pathology, University of Benin Teaching Hospital, Benin City, Nigeria

Email address:

oliviadynski@yahoo.com (E. O. Ogboghodo)

${ }^{*}$ Corresponding author

\section{To cite this article:}

Esohe Olivia Ogboghodo, Joy Chinyere Nwaogwugwu, Otaniyenuwa Eloghosa Obarisiagbon, Orezimena Temitope Omo-Ikirodah, Edith Imuwahen Uwugiaren, Obehi Aituaje Akoria, Darlington Ewaen Obaseki, Mokogwu Ndubuisi. Health Facility Preparedness and Response to COVID-19: An Assessment of Employee Satisfaction in a Teaching Hospital in Southern Nigeria. Central African Journal of Public Health. Vol. 6, No. 5, 2020, pp. 242-250. doi: 10.11648/j.cajph.20200605.12

Received: August 4, 2020; Accepted: August 21, 2020; Published: September 10, 2020

\begin{abstract}
Employee satisfaction is increasingly recognized as a measure that largely determines the productivity and efficiency of health institutions. This study assessed employees' satisfaction with preparedness for, and response to COVID-19 in a tertiary hospital in Benin City, Edo State, Nigeria. This was a descriptive cross-sectional study using mixed methods (qualitative and quantitative) for data collection from employees at the University of Benin Teaching Hospital, Benin City. Quantitative data were analyzed using IBM SPSS version 25.0 while qualitative data were analyzed thematically using ATLAS. ti software. The statistical measures for the quantitative analysis were the adjusted odds ratios (OR) and 95\% confidence intervals $(\mathrm{CI})$. The level of significance was set at $\mathrm{p}<0.05$. A total of 590 respondents with mean age $38.9 \pm 9.7$ years and 20 focus group discussion (FGD) participants participated in the study. Overall, $368(62.4 \%)$ of employees were satisfied with the measures put in place to combat COVID-19 in the facility. Staff were most satisfied with infection prevention and control measures (72.9\%) and information and education communication (IEC) on respiratory hygiene and cough etiquette (67.8\%). Age of respondents $<40$ years, being a clinical staff and having direct contact with patients were significant predictors of employee satisfaction, aOR 0.661 ( $\mathrm{p}=0.024)$; aOR 0.528 ; $(\mathrm{p}=0.003)$; aOR $0.594(\mathrm{p}=0.039)$, respectively. Thematic analysis of the FGDs revealed that awareness creation about COVID-19, and policies and protocols were areas that employees were least satisfied with. In conclusion, two-thirds of employees were overall satisfied with measures put in place to combat COVID-19 in the health facility. Younger staff and those who worked in clinical areas, were less satisfied than other groups. The management of the study facility can leverage these findings to sustain and strengthen IPC and IEC measures while addressing communication of policies and protocols in areas where staff were less satisfied.
\end{abstract}

Keywords: Employee Satisfaction, Tertiary Health Facilities, COVID-19

\section{Introduction}

Employee satisfaction is the mental feeling of favorableness which an individual has about his job. It influences the productivity of the employee in the workplace. Most successful organizations consider employee satisfaction to be vital for work performance. It has its impact on the general life of the employees as a satisfied employee is 
contented, and has a better physical and mental well-being. This has led to the general saying that "a happy employee is a productive employee." [1, 2] As such, organizations experience increased productivity and huge success whenever management actively works to improve attitudes, quality of work-life and job satisfaction of employees. [2, 3]

The workforce is central to advancing health in every health system. Health workers are the backbone of all health care systems needed for effective provision of health care, management of health programs and response to health emergencies. [4] A strong health workforce is an integral part of every resilient health system and during a pandemic, it is an essential foundation for the recovery of our societies, economies and preparedness for future health emergencies. [4] Therefore, the presence of high-quality motivated staff is a key aspect of health system performance. [3]

Job satisfaction among healthcare workers is increasingly being recognized as a measure that should be included in quality improvement programs in health care delivery as it largely determines the productivity and the efficiency of health institutions. Although efficient health service delivery is affected by many factors such as human resources and health infrastructure, human resources are a vital component and the most valuable asset for delivering efficient and sustainable health services. [5-7]

Hospitals play a critical role within the health system in providing essential medical care to the community, especially in a pandemic. [7] Effective pandemic preparedness and response requires the engagement of health care facilities; and healthcare workers will need to be prepared and trained to meet the increased demands. [7, 8] Healthcare facilities must be prepared to adjust to varying stressors on the system over time through collaboration with diverse partners, effective information sharing and coordination of response activities. [8, 9]

More than any other group of workers, health workers are disproportionally at risk of infection and death as they are at the frontlines of the COVID-19 outbreak response. Currently in Nigeria (as of June 3, 2020) 812 healthcare workers have been infected with COVID-19. [10] With increased COVID19 infection worldwide, health systems especially in lowincome countries, face challenges in providing adequate protective equipment and infection prevention measures to protect their workforce. Inability to protect healthcare workers (HCWs) will not only lead to needless loss of lives but also deplete already stretched health systems, thereby depriving these health systems of a crucial asset to fight the current pandemic. [9]

The occupational health and safety of healthcare workers are fundamental to enabling them perform optimally during a pandemic crisis. Achieving healthcare worker protection should therefore be a priority to any hospital management. As employers of labor, managers of health facilities should assume the overall responsibility of ensuring that all necessary preventive and protective measures are taken to ensure occupational safety and minimize health risks. [11] Information on the transmission of the disease should be shared with health workers as widely and as quickly as possible, including information on the most recent guidelines, measures to prevent contagion, and how they should be implemented. [11, 12]

Dialogue between health workers and employers ensure that policies and procedures are implemented in an appropriate manner. The availability of Personal Protective Equipment (PPE) is also critical, in addition to training and education on how to use such equipment correctly. In addition, testing for COVID-19 infection should be made available for health workers as widely as possible in order to ensure both workers' health and patients' safety. [11-13] These practices will reduce the risk of infection and transmission of the disease and positively influence HCWs job satisfaction and dedication. [13]

This study was conducted to assess the level of satisfaction of employees in University of Benin Teaching Hospital with the hospital's response to the COVID-19 pandemic. Findings from this study will add to the existing body of knowledge and provide the hospital's management and other managers especially in resource-poor settings - information on what aspects of the pandemic preparedness and response to strengthen and/or further address.

\section{Methods}

\subsection{Setting}

This study was conducted in the University of Benin Teaching Hospital (UBTH), Benin-City, and utilized a descriptive cross-sectional study design with mixed methods (quantitative and qualitative) data collection. UBTH is one of the three public hospitals in Edo State designated by the Government as an isolation facility for the management of COVID-19 patients, and the only isolation center with Intensive Care Unit (ICU) capacity in the State. [14] UBTH currently has a staff strength of 3,840 employees and renders promotive, preventive, curative and rehabilitative services in various departments. These departments include; Internal Medicine, Surgery, Pediatrics, Mental Health, Community Health, Obstetrics and Gynecology, Radiology, Ear, Nose and Throat, Anesthesiology, Ophthalmology, Family Medicine, Dentistry, Physiotherapy and Occupational Therapy.

At the start of the pandemic in Nigeria, the management of UBTH constituted a COVID-19 Response Team with seven pillars viz: Clinical Management, Diagnostic, Logistics and Supplies, Infection Prevention and Control/Surveillance, Screening and triage, Risk Communication, and Central Governance/Research. Each pillar comprised pillar heads and members, with a mandate to effectively see to the coordinated response to COVID-19 in the facility. Strategic meetings were held twice weekly for monitoring and evaluation of the response. The isolation facility located in the hospital comprised a 24-bed isolation ward, an 18-bed isolation annex, a 2- bed Intensive Care Unit, and an 18-bed holding bay for suspected COVID-19 cases. Management of COVID 19 patients was carried out by a Rapid Response 
Team (RRT) made up of multidisciplinary personnel. Within the hospital, management ensured that facilities and equipment necessary for standard precautions and transmission-based precautions were made available to all staff. Signage and Information Education and Communication (IEC) posters on COVID-19 were placed at strategic locations, and advisories were regularly communicated to staff. The Hospital also adopted and enforced the "No face mask No entry" policy to ensure the mitigation of the spread of COVID -19 by staff and visitors within the facility.

\subsection{Sampling}

The study population comprised all cadres of employees who had been in the facilities' employ for at least six months prior to the survey in order to provide an objective assessment of the health workers' satisfaction. A minimum sample size of 583 was calculated using the appropriate formula for a single proportion. [15] This was calculated considering a standard normal deviate of 1.96 at a significance level of $5 \% ; 64.8 \%$ (the prevalence of health workers who were satisfied with their jobs as reported in a 2019 study and a $10 \%$ attrition rate (nonresponse). [9] A stratified sampling technique was employed in selecting healthcare workers for this study. The occupation of the employees formed the basis of each stratum. Proportional allocation was used to determine the number of employees in each department. From each department, a systematic sampling technique was used to select the respondents. Using the list of workers in each department as the sampling frame, a sampling interval was calculated. The first respondent was selected using simple random sampling method, after which every nth respondent was selected until the required sample size was obtained.

\subsection{Data Collection and Analysis}

A combination of quantitative and qualitative data collection tools were used to collect data. The quantitative tool was a structured questionnaire divided into sections to assess satisfaction in six domains viz: 1. Awareness creation and health education; 2. Availability of PPEs; 3. Infection prevention and control (IPC) measures; 4. Screening and triaging of patients; 5. Work environment and 6. Policies and protocols. There were a total of 35 questions in these 6 domains: awareness creation and health education (5 questions); availability of PPEs (7 questions); IPC measures (9 questions); screening and triage (6 questions); work environment (4 questions); policies and protocols (4 questions).

The qualitative tool was a Focused Group Discussion (FGD) guide designed in line with the specific objectives, to give respondents opportunities to share their experiences without inhibitions and give clarity to the responses obtained in the quantitative data.

Four research assistants were trained for two days on data collection to enhance the validity and repeatability of the research tools prior to the survey. Questionnaires were self- administered and collected from respondents at their convenience. Two Focus Group Discussions (FGDs) involving 11 and 9 participants respectively, categorized based on occupation were carried out with questions targeted at assessing employee satisfaction with the hospital management's response to COVID-19. The FGDs took place at a venue that was convenient for the participants, within the facility. Participants were briefed on the purpose of the discussion and were encouraged to freely share their views. The discussion was guided by one of the researchers such that the discussion was not dominated by a set of participants. Notes were taken during the discussion and audio recording was also done. The recordings were subsequently transcribed to provide information that was inadvertently missed during note-taking. The discussion lasted for one hour, at the end of which participants were verbally appreciated for their participation.

Quantitative data was screened for completeness, coded and analyzed using IBM-SPSS version 25.0. Employee satisfaction was assessed using a 3-point Likert scale. The most favorable answer was given a score of 2 and the least favorable answer was given a score of 0 giving a maximum score of 70 and a minimum score of 0 . Overall satisfaction score was computed by converting to percentages and grouped as not satisfied $(<60 \%)$ and satisfied $(\geq 60 \%)$.

The statistical measure for the quantitative analysis was the adjusted odds ratio (OR) and 95\% confidence interval (CI). The level of significance was set at $\mathrm{p}<0.05$ for all statistical associations. The results are presented in frequency tables.

Qualitative data from the FGD were analyzed using the ATLAS ti Vs. 6.0. Data were sorted and classified according to research objectives. Themes were interpreted and elaborated on to provide descriptive narratives of the findings in line with the study objectives.

\subsection{Ethics}

Ethical approval was obtained from the Ethics and Research Committee of University of Benin Teaching Hospital. Permission to conduct the study was also obtained from the hospital management. Informed consent was obtained from each respondent after the purpose of the study had been explained by the researcher. All aspects of the study, including the aim, interview procedures, anticipated benefits and potential hazards were explained to the respondents.

Participation in the study was voluntary and participants were informed of their right to decline participation or to withdraw from the study at any time if they so desired, with no penalties or loss of benefits. Confidentiality and privacy were guaranteed, and questionnaires were anonymized.

\section{Results}

A total 590 respondents with mean age $38.9 \pm 9.7$ years participated in the quantitative study while the FGD comprised a total of 20 participants (13 females and 7 males) with a mean age of $37.4 \pm 6.3$ years. Female respondents 
[384 (65.1\%)] made up two-thirds of the study population, majority of whom were married [428 (72.5\%)]. Most respondents [556 (94.2\%)] had completed tertiary education. A large proportion [217 (36.8\%)] of the respondents were nurses, followed by administration staff [166 (28.1\%)] and doctors [101 (17.1\%)]. Majority [395 (66.9\%)] of the respondents worked as clinical staff; 195 (33.1\%) were nonclinical. (Table 1). Ninety respondents $(15.2 \%)$ had been in the employ of the hospital for over 20 years, 148 (25.1\%) for 10 to 19 years, and $352(59.7 \%)$ for less than 10 years, at the time of the survey (Table 1).

Table 1. Socio-Demographic and Occupational Characteristics of Respondents.

\begin{tabular}{|c|c|c|}
\hline Variables & Frequency $(n=590)$ & Percent \\
\hline \multicolumn{3}{|c|}{ Age group (years) } \\
\hline $20-29$ & 112 & 19.0 \\
\hline $30-39$ & 204 & 34.6 \\
\hline $40-49$ & 166 & 28.1 \\
\hline $50-59$ & 106 & 18.0 \\
\hline $60-69$ & 2 & 0.3 \\
\hline Mean \pm SD & $38.9 \pm 9.7$ & \\
\hline \multicolumn{3}{|l|}{ Sex } \\
\hline Female & 384 & 65.1 \\
\hline Male & 206 & 34.9 \\
\hline \multicolumn{3}{|l|}{ Religion } \\
\hline Christian & 585 & 99.2 \\
\hline Muslim & 5 & 0.8 \\
\hline \multicolumn{3}{|c|}{ Highest level of education } \\
\hline Primary & 8 & 1.4 \\
\hline Secondary & 26 & 4.4 \\
\hline Tertiary & 556 & 94.2 \\
\hline \multicolumn{3}{|l|}{ Occupation } \\
\hline Nurse & 217 & 36.8 \\
\hline Administration & 166 & 28.1 \\
\hline Doctor & 101 & 17.1 \\
\hline Lab scientist & 39 & 6.6 \\
\hline Pharmacist & 23 & 3.9 \\
\hline Health assistant & 20 & 3.4 \\
\hline Cleaner & 13 & 2.2 \\
\hline Paramedic & 5 & 0.8 \\
\hline Physiotherapy & 4 & 0.7 \\
\hline Optometry & 2 & 0.3 \\
\hline \multicolumn{3}{|c|}{ Years in employment } \\
\hline$<10$ & 352 & 59.7 \\
\hline $10-19$ & 148 & 25.1 \\
\hline $20-29$ & 75 & 12.7 \\
\hline $30-39$ & 15 & 2.5 \\
\hline \multicolumn{3}{|l|}{ Class of staff } \\
\hline Clinical & 395 & 669. \\
\hline Non-Clinical & 195 & 33.1 \\
\hline \multicolumn{3}{|c|}{ Direct patient contact } \\
\hline Yes & 467 & 79.2 \\
\hline No & 123 & 20.8 \\
\hline
\end{tabular}

Regarding awareness creation and health education, about two-thirds [400 (67.8\%)] of respondents were satisfied with the availability of IEC materials on respiratory hygiene and cough etiquette within the facility. Over half [313 (53.1\%)] were satisfied with the level of education that had been given on hand hygiene. However, only a third [249 (42.2\%)] expressed satisfaction with training on the use of PPEs (Table 2).

The FGDs revealed that the HCWs believed that management could do more on awareness creation and health education.

Management needs to improve more on awareness creation especially among non-clinical staff. Only the doctors and nurses seem to be well informed on the mode of transmission and prevention of COVID-19. The non-clinical staffs just wear these PPEs but don't really understand their purpose. A. O. M/ 35 years (medical doctor).

In my opinion Management has done great so far in the area of awareness creation of COVID-19. I. G. Male/36 years (nurse).

Other respondents opined that posters in local languages should be available within the facility and social media engagement should be more visible.

The management can do better with awareness creation. At times like this, banners and posters on COVID-19 written in local languages should be available in the facility. The Hospital has an active social media account yet awareness on those pages and, information on prevention and transmission of COVID-19 is minimal. UBTH ought to be at the forefront of creating awareness both to its staff and the general public. O. E. Male/35 years (medical doctor).

Regarding availability of PPEs, about two-thirds of the respondents $[378(64.1 \%)]$ were satisfied with the availability of face masks in the facility and $334(56.6 \%)$ were satisfied with the provision of goggles. More than one third [223 $(37.8 \%)]$ of respondents were satisfied with the availability of latex gloves and about one fifth [130 (22.0\%)] were satisfied with the availability of face shields (Table 2).

The FDGs clarified respondents' dissatisfaction with availability of specific PPEs such as face masks and face shields. These PPEs were said to be few in the wards and had to be rationed.

Management has tried to an extent in the provision of PPEs but not all PPEs are provided. Face shield weren't previously available until recently. We had to purchase our own personal face shields ourselves. A. N Female/34years (medical doctor)

Majority [458 $(77.6 \%)]$ of the respondents were satisfied with the availability of hand sanitizers and hand dryers within the facility. Over half [331 (56.1\%)] were satisfied with the availability of hand washing stations at various points of care and $319(54.1 \%)$ were satisfied with the numbers of linen bags available to them (Table 2).

Respondents in the FGDs were particularly satisfied with the availability of running water, liquid soap and dryers but reported that waste bins were not correctly color coded and linen bags were not sufficient.

Management has tried in the provision of hand washing points because there is running water and soap at every corner so I think I will give management an $80 \%$. E. I. Male/32 years (medical doctor).

Waste bins are not appropriately color coded. Sometimes you go to the wards and there are only black lined bins and other times red or yellow lined bins. It is the colour provided by the stores that is used in the wards. E. G. Female/40 years (nurse).

Concerning screening and triaging, $369(60.0 \%)$ of the 
respondents reported being satisfied with the number of digital thermometers provided for screening of patients. More than half $[332(56.3 \%)]$ were also satisfied with the ongoing triage processes at different points of entry into the emergency departments and clinics (Table 2).

Participants in the FGDs were not satisfied with the implementation of social distancing rule in patient waiting areas, as patients were seen to crowd themselves in the waiting area. Others opined that social distancing was also necessary among in-patients in the wards.

Social distancing is only being implemented in the consulting rooms but not in patients' waiting area and this defeats the purpose. Canopies should be created outside with space enough to allow for social distancing. There should also be stickers on the ground to measure and show the distance for proper social distancing. O. D Male/32 years (medical doctor).

A way to reducing crowding of patients in waiting areas is to book patients per time so not all patients who are to see the doctor are together at the same time. O. J. Male/35 years (medical doctor).

Regarding the work environment domain, 202 (34.2\%) of the respondents were satisfied with the regular updates provided to the staff. Similarly, 199 (33.7\%) respondents were satisfied with the release of work advisories about COVID-19. Concerning policies and protocols, less than one third $[184(31.2 \%)]$ of employees were satisfied with the protocols and documents on case management (Table 2).

The FGDs provided more depth about employees' dissatisfaction with clinical management and testing protocols released to staff:

There are no regular updates on COVI-19 provided to the staff. We are not aware of the protocol of diagnosis neither are we aware of how long it takes for a result to come out. I am not aware of the number of infected patients in the isolation wards or how long it takes for a COVID-19 test to result to come out. E. O. Male/35 years (medical doctor).

Enforcement of the use of face masks by patients and relatives is weak. Even the security men at the gate do not wear theirs all the time so how can they enforce it among those coming into the hospital? N. E. Female/35 years (nurse).

Assessing the employee satisfaction domains, 389 (65.9\%) of respondents were satisfied with the awareness creation and health education carried out in the facility; 257 (43.6\%) were satisfied with the supply of PPEs; $430(72.9 \%)$ were satisfied with the infection prevention measures put in place; 324 $(54.9 \%)$ were satisfied with the screening and triage processes; 219 (37.1\%) were satisfied with the current work environment and $239(40.5 \%)$ satisfied with policies and protocols in place to guard against COVID-19.

Table 2. Staff Satisfaction Domains.

\begin{tabular}{|c|c|c|c|}
\hline Variable & Satisfied n (\%) & Undecided n (\%) & Unsatisfied n (\%) \\
\hline \multicolumn{4}{|l|}{ Awareness creation \& health education } \\
\hline Presence of flyers and posters on respiratory hygiene and cough etiquette & $400(67.8)$ & $80(13.6)$ & $110(18.6)$ \\
\hline Adequate education on hand hygiene, respiratory etiquette and standard precautions & $313(53.1)$ & $105(17.8)$ & $172(29.2)$ \\
\hline Presence of flyers and posters on hand washing within the facility & $291(49.3)$ & $123(20.8)$ & $176(29.8)$ \\
\hline Adequate training on appropriate use of PPEs & $249(42.2)$ & $140(23.7)$ & $201(34.1)$ \\
\hline \multicolumn{4}{|l|}{ Availability of PPEs } \\
\hline Availability of goggles & $334(56.6)$ & $57(9.7)$ & $199(33.7)$ \\
\hline Availability of Hazmat suits & $297(50.3)$ & $64(10.8)$ & $229(38.8)$ \\
\hline Availability of coveralls & $283(48.0)$ & $62(10.5)$ & $245(41.5)$ \\
\hline Availability of latex disposable gloves & $223(37.8)$ & $74(12.5)$ & $293(49.7)$ \\
\hline Availability of face shields & $130(22.0)$ & $79(13.4)$ & $381(64.6)$ \\
\hline Availability of boots & $122(20.7)$ & $91(15.4)$ & $377(63.9)$ \\
\hline \multicolumn{4}{|l|}{ Infection prevention and control } \\
\hline Availability of hand dryers & $458(77.6)$ & $47(8.0)$ & $85(14.4)$ \\
\hline Availability of high level disinfection for contaminated areas & $410(69.5)$ & $63(10.7)$ & $117(19.8)$ \\
\hline Availability of cleaning and disinfecting agents for all frequently touched surfaces & $369(62.5)$ & $69(11.7)$ & $152(25.8)$ \\
\hline Availability of colour coded waste bins at designated points & $355(60.2)$ & $90(15.3)$ & $145(24.6)$ \\
\hline Availability of hand washing stations at points of care & $331(56.1)$ & $62(10.5)$ & $197(33.4)$ \\
\hline Availability of liquid soap & $270(45.8)$ & $77(13.1)$ & $243(41.2)$ \\
\hline Availability of clean running water & $247(41.9)$ & $79(13.4)$ & $264(44.7)$ \\
\hline Availability of linen bags & $319(54.1)$ & $123(20.8)$ & $148(25.1)$ \\
\hline
\end{tabular}

Table 2. Contd: Staff Satisfaction Domains.

\begin{tabular}{|c|c|c|c|}
\hline Variable & Satisfied n (\%) & Undecided n (\%) & Unsatisfied n (\%) \\
\hline \multicolumn{4}{|l|}{ Screening and triaging } \\
\hline Availability of sufficient quantity of digital thermometers & $369(62.5)$ & $86(14.6)$ & $150(25.4)$ \\
\hline Triage at points of entry into the emergency departments and clinics & $332(56.3)$ & $108(18.3)$ & $150(25.4)$ \\
\hline Implementation of social distancing rule in patient waiting areas & $283(48.0)$ & $128(21.7)$ & $179(30.3)$ \\
\hline Availability of sufficient holding areas for suspected cases & $250(42.4)$ & $158(26.8)$ & $182(30.8)$ \\
\hline Availability of sufficient isolation wards for confirmed cases & $240(40.7)$ & $132(22.4)$ & $218(36.9)$ \\
\hline
\end{tabular}




\begin{tabular}{|c|c|c|c|}
\hline Variable & Satisfied n (\%) & Undecided n (\%) & Unsatisfied n (\%) \\
\hline $\begin{array}{l}\text { Availability of ventilators for management of critical cases } \\
\text { Work Environment }\end{array}$ & $181(30.7)$ & $173(29.3)$ & $236(40.0)$ \\
\hline Regular update/communication with staff & $202(34.2)$ & $165(28.0)$ & $223(37.8)$ \\
\hline Release of work place advisories as regards COVID-19 & $199(33.7)$ & $154(26.1)$ & $237(40.2)$ \\
\hline Staff-risk assessment and re-deployment of vulnerable groups & $187(31.7)$ & $175(29.7)$ & $228(38.6)$ \\
\hline Provision of incentives for frontline staff & $179(30.3)$ & $135(22.9)$ & $237(40.2)$ \\
\hline \multicolumn{4}{|l|}{ Policies and protocols } \\
\hline Protocols and documents for case management & $184(31.2)$ & $209(35.4)$ & $184(31.2)$ \\
\hline Protocols and documents for specimen collection and handling & $174(29.5)$ & $255(43.2)$ & $161(27.3)$ \\
\hline Protocols and documents for handling of corpses & $165(28.0)$ & $272(46.1)$ & $153(25.9)$ \\
\hline
\end{tabular}

Overall, $368(62.4 \%)$ respondents were satisfied with the measures put in place to address COVID-19 in the study facility (Table 3).

Table 3. Staff satisfaction with preparation for and response to COVID-19 in UBTH.

\begin{tabular}{lll}
\hline Variable & $\begin{array}{l}\text { Satisfied } \\
\text { (Percent) }\end{array}$ & $\begin{array}{l}\text { Unsatisfied } \\
\text { (Percent) }\end{array}$ \\
\hline Awareness creation \& health education & $389(65.9)$ & $201(34.1)$ \\
Availability of PPEs & $257(43.6)$ & $333(56.4)$ \\
Infection prevention and control & $430(72.9)$ & $160(27.1)$ \\
Screening and triaging & $324(54.9)$ & $266(45.1)$ \\
Work environment & $219(37.1)$ & $371(62.9)$ \\
Policies and protocols & $239(40.5)$ & $351(59.5)$ \\
Overall satisfaction & $368(62.4)$ & $222(37.6)$ \\
\hline
\end{tabular}

The sex and religion of respondents were not found to be significant predictors of satisfaction. Age of respondents and having a direct contact with a patient diagnosed with COVID-19 were significantly associated with satisfaction. In the multivariate regression analysis, respondents who were younger than 40 years were $66 \%$ less likely to be satisfied than those aged 40 years and above (adjusted OR 0.661; CI 0.461- 0.947; $\mathrm{p}=0.024$ ).

Clinical staff were $53 \%$ less likely to be satisfied with the COVID-19 measures put in place in the facility compared to their non-clinical counterparts (adjusted OR: 0.528; CI 0.347$0.803 ; \mathrm{P}=0.003$ ). Respondents who had direct contact with patients were $59 \%$ less likely to be satisfied than those who did not (adjusted OR 0.594; CI 0.363-0.973; p=0.038 - Table 4).

Table 4. Unadjusted and adjusted predictors of staff satisfaction.

\begin{tabular}{|c|c|c|c|c|}
\hline Predictors & Unadjusted OR (95\% CI) & p-value & Adjusted OR (95\% CI) & p-value \\
\hline \multicolumn{5}{|c|}{ Age group (years) } \\
\hline$<40$ & $1.662(1.175-2.350)$ & 0.004 & $0.661(0.461-0.947)$ & 0.024 \\
\hline$\geq 40 *$ & 1 & & 1 & \\
\hline \multicolumn{5}{|l|}{ Sex } \\
\hline Male & $1.291(0.913-1.826)$ & 0.149 & $0.726(0.502-1.050)$ & 0.089 \\
\hline Female* & 1 & & 1 & \\
\hline \multicolumn{5}{|l|}{ Religion } \\
\hline Christian & $0.599(0.148-2.419)$ & 0.467 & $2.142(0.511-8.974)$ & 0.297 \\
\hline Muslim* & 1 & & 1 & \\
\hline \multicolumn{5}{|c|}{ Occupation group } \\
\hline Clinical & $2.140(1.469-3.119)$ & $<0.001$ & $0.528(0.347-0.803)$ & 0.003 \\
\hline Non-clinical & 1 & & 1 & \\
\hline \multicolumn{5}{|c|}{ Direct patient contact } \\
\hline Yes & $2.164(1.379-3.398)$ & 0.001 & $0.594(0.363-0.973)$ & 0.039 \\
\hline No* & 1 & & 1 & \\
\hline
\end{tabular}

\section{Discussion}

Employee satisfaction is pivotal to the well-being of any organization. This can be seen in employees who find their jobs fulfilling and enjoyable, and will thus accord the job the deserved importance. This is particularly important in crisis situations, e.g. during a pandemic. This study recorded the highest employee satisfaction with COVID-19 preparedness in the infection prevention control (IPC) measures put in place by the management. IPC measures are important as they have been found to reduce the transmission of infections especially within hospital settings. Adequate IPC measures such as sufficient hand washing points and the availability of PPES can lead to decreased transmission of COVID-19, directly improving $\mathrm{HCWs}$ ' satisfaction and increasing productivity. [16]

Respondents were mostly satisfied with the availability of hand sanitizers and automated hand dryers, a finding that was corroborated in the FGDs where participants expressed their satisfaction with the availability of hand sanitizers, running water, liquid soap and dryers. Hand hygiene is recognized globally as a leading measure of IPC and has been shown to be effective in decreasing the transmission of common respiratory viruses, including human coronaviruses. [16] Findings from a study done in China to assess healthcare staff's conditions and job satisfaction and their associated predictors during the epidemic peak of COVID-19 revealed that PPE access predicted better physical health and job satisfaction, demonstrating its importance beyond physical protection. [17]

Healthcare workers (HCWs) play an essential role in 
containing the ongoing COVID-19 outbreak and alleviating the increasing infection risk. They rely on personal protective equipment in addition to other IPC practices to protect themselves and their patients from being infected and infecting others. [18] Less than half of the respondents were satisfied with the availability of PPEs. This was corroborated by findings from the FGDs which revealed participants' dissatisfaction with availability of specific PPEs like coveralls and face shields. This is not unusual in a resourceconstrained setting, as even developed countries have had challenges of PPE unavailability. The World Health Organization had previously warned that severe and mounting disruption to the global supply of personal protective equipment (PPE) would occur due to rising demand, panic buying, hoarding and misuse. [19] These reasons could account for the reduced availability of some PPEs in this tertiary institution.

Inadequate supply of PPEs can leave HCWs dangerously ill-equipped to care for COVID-19 patients, thereby putting them at risk of infection, and hindering the prevention and control of COVID-19. [18] Infected HCWs reduce the workforce of well-trained HCWs that are able to provide patient care, which in turn reduces the hospitals' ability to deal with the COVID-19 outbreak. Furthermore, reduced satisfaction with IPC measures in place at the hospital could lead to poor work attitude, with negative impacts on the quality of patient care. [16] Managements of tertiary institutions can increase the local production of some of these PPEs like face shields and coveralls to improve availability.

Although majority of the respondents were satisfied with management's efforts at awareness creation among staff, employees were specifically not satisfied with the training on the use of PPEs. The FGD revealed varied and diverse perceptions of respondents in this regard, as majority of the participants opined that there was more to be done on awareness creation especially among non-medical personnel and the general public. Awareness creation and health education of HCWs is important for creating prevention beliefs, forming positive attitudes and promoting positive behaviors. These in turn influence job satisfaction. [20]

Healthcare workers can pose a transmission risk when they are not adequately trained on proper donning and use of PPEs Although healthcare workers carry a significant burden of coronavirus infections worldwide, the rates can be lowered with the use of personal protective equipment combined with proper training in infection control. [21] It is therefore recommended that managements of health facilities frequently organize continuous medical education and training for easy and targeted dissemination of information to different categories of staff.

Respondents were dissatisfied with the frequency of communication and release of work place advisories regarding COVID-19 by the management. Employee dissatisfaction has negative consequences on the structure and work flows of organizations. These consequences include greater non-conformance with procedures and policies, increase in work accidents, and organizational conflicts that may increase the rate of medical errors, and jeopardize patient safety. [22] Management can improve on communicating with health staff by taking advantage of digital media platforms for quick dissemination of information.

More than half of the respondents were satisfied with the screening and triage of patients with respect to COVID-19, but the FGDs revealed that participants were particularly not satisfied with the implementation of the social distancing rule in patient waiting areas and wards. Some participants also opined that holding areas for suspected cases were inadequate, as some suspected cases were being nursed in the wards. Early isolation of suspected cases in the hospital is important to reduce the likelihood of nosocomial spread. However, in the ongoing COVID-19 pandemic, patients with COVID-19 may present with respiratory syndromes similar to those caused by common respiratory viruses, and early isolation and containment could be challenging especially during significant ongoing community transmission. [23] Undiagnosed cases of COVID-19 in the wards and clinics may share common facilities. Such social mingling can represent a potential route for transmission of COVID-19 within the hospital, underscoring the value of social distancing, which has been identified as crucial for containment of the COVID-19 pandemic in hospitals as well as in the community. [23]

Only one third of employees were satisfied with the hospital's policies and protocols regarding case management of COVID-19. This was mirrored in the responses of the FGD participants, some of who said that they were unaware of the protocols regarding testing and diagnosis of COVID19. Healthcare workers (HCWs) are at the front lines of the COVID-19 pandemic response and are at risk of pathogen exposure. A poor understanding of the disease among HCWs can result in delayed identification and treatment, leading to rapid spread of infections. As an emerging, rapidly changing global health challenge that is affecting all sectors, [24] it is of paramount importance that HCWs across the world have adequate knowledge about all aspects of COVID-19 - its clinical manifestation, diagnosis, proposed treatment, and established prevention strategies. [24]

Overall, most of the respondents interviewed were satisfied with the measures put in place in response to COVID-19 in the study facility. This is a key finding, as highly satisfied healthcare staff are more likely to provide higher quality medical services, resulting in better healthcare outcomes and higher patient satisfaction. $[25,26]$ We recommend that these measures should be sustained, while concerted efforts should be made to address those aspects of the response to COVID-19 with which the staff were not so satisfied.

\section{Conclusion}

This study revealed that staff satisfaction was highest with IPC measures, and information and education materials on respiratory hygiene and cough etiquette and lowest as regards work environment and studies and protocols. The 
management of the study facility can leverage on these findings to sustain and strengthen IPC and awareness creation measures, while addressing aspects of the COVIDresponse such as provision of PPEs and communication of policies and protocols, with which staff were dissatisfied.

\section{Ethical Conformity Statement}

Ethical clearance to conduct this research was obtained from the Department of Community Health, University of Benin.

\section{Conflict of Interests}

All the authors do not have any possible conflict of interest.

\section{Acknowledgements}

The authors wish to acknowledge the management and staff of the University of Benin Teaching Hospital.

\section{References}

[1] Janardhanan A. K and George S. A Study On Job Satisfaction Of Employees In Bpcl - Kochi Refinery Limited, Ambalamugal. 2011. Available at: https://www.researchgate.net/publication/281857611_A_STU DY_ON_JOB_SATISFACTION_OF_EMPLOYEES_IN_BP CL-KOCHI_REFINERY_LIMITED_AMBALAMUGAL_

[2] Singh T, Kaur M, Verma M and Kumar R. Job satisfaction among health care providers: A cross-sectional study in public health facilities of Punjab, India. Journal of family Medicine and Primary Care. 2019; 8 (10): 3268-3275. Available at: http://www.jfmpc.com/article.asp?issn=2249-4863;

year $=2019$; volume $=8$; issue $=10 ;$ spage $=3268$; epage $=3275$; aulast $=$ Singh

[3] Ndubuisi-Okolo P. U, Yusuf A. E and Anigbogu T. Influencing Employees Job Attitude In Nigeria: A Critical Review. IOSR Journal of Business and Management. 2017: 19 (12): 35-40. Available at: https://www.researchgate.net/publication/322702272_Influenc ing_Employees_Job_Attitude_In_Nigeria_A_Critical_Review.

[4] Peters, D. H., Chakraborty, S., Mahapatra, P. et al. Job satisfaction and motivation of health workers in public and private sectors: cross-sectional analysis from two Indian states. Hum Resour Health. 2010; 8 (27). Available at: https://doi.org/10.1186/1478-4491-8-27.

[5] Merga, H., Fufa, T. Impacts of working environment and benefits packages on the health professionals' job satisfaction in selected public health facilities in eastern Ethiopia: using principal component analysis. BMC Health Serv Res. 2019; 19 (494). Available at: https://doi.org/10.1186/s12913-0194317-5.

[6] Deriba BK, Sinke SO, Ereso BM, Badacho AS. Health professionals' job satisfaction and associated factors at public health centers in West Ethiopia. Hum Resour Health. 2017; 15 (1): 36. Available at: doi: 10.1186/s12960-017-0206-3 .

[7] Center for Disease Control and Prevention. Healthcare System Preparedness and Response. CDC. 2016. Available at: https://www.cdc.gov/flu/pandemic-resources/planningpreparedness/healthcare-preparedness-response.html.

[8] World Health Organisation. Coronavirus disease (COVID-19) outbreak: rights, roles and responsibilities of health workers, including key considerations for occupational safety and health. WHO. 2020. Available at: https://www.who.int/emergencies/diseases/novel-coronavirus2019/technical-guidance/health-workers.

[9] Gyang M. D, Dankyau M, Gidado S et al. Job satisfaction among Healthcare Workers at a District Hospital in Plateau State, Nigeria. Journal of Medicine in the Tropics. 2020; 20: 111-116. Available at: https://www.researchgate.net/publication/334487548_job_sati sfaction_among_healthcare_workers_at_a_district_hospital_in _plateau_state_nigeria.

[10] Tih. F. Nigeria: 800 Healthcare Workers Infected with Covid19. AA. 2020. Available at: https://www.aa.com.tr/en/africa/nigeria-800-health-workersinfected-with-covid-19/1863747.

[11] Wiskow C. and Hopfe M. Five ways to protect health workers during the COVID-19 crisis. ILO. 2020. Available at: https://iloblog.org/2020/04/01/five-ways-to-protect-healthworkers-during-the-covid-19-crisis/.

[12] World Health Organisation. Strengthening the Health Systems Response to COVID-19. WHO. 2020. Available at: http://www.euro.who.int/_data/assets/pdf_file/0007/436354/s trengthening-health-systems-response-COVID-19-technicalguidance-1.pdf.

[13] Center for Disease Prevention and Control. Steps Healthcare Facilities Can Take Now to Prepare for COVID-19. CDC. 2020. Available at: https://www.cdc.gov/coronavirus/2019ncov/hcp/steps-to-prepare.html.

[14] University of Benin Teaching Hospital. About UBTH. UBTH. 2020. Available at: https://ubth.org/general-information/.

[15] Cochrane G. Sampling techniques. 2nd ed. New York.: John Willey and Sons Inc; 1963.

[16] Peters A, Lotfinejad N, Simniceanu A, Pittet D. The economics of infection prevention: why it is crucial to invest in hand hygiene and nurses during the novel coronavirus $J$ Infect. 2020; S0163-4453 (20) 302401. Available at: https://www.ncbi.nlm.nih.gov/pmc/articles/PMC7179502/.

[17] Zhang S, Liu J, Afshar A, Nawaser K, Yousefi A, Li J and Sun, S. At the height of the storm: Healthcare staff's health conditions and job satisfaction and their associated predictors during the epidemic peak of COVID-19. 2020; 87: 144-146. Available at: https://www.sciencedirect.com/science/article/pii/S088915912 0307832 .

[18] Lai, X., Wang, X., Yang, Q. et al. Will healthcare workers improve infection prevention and control behaviors as COVID-19 risk emerges and increases, in China? Antimicrob Resist Infect Control. 2020; 9 (83). Available at: https://doi.org/10.1186/s13756-020-00746-1.

[19] WHO. Shortage of personal protective equipment endangering health workers worldwide. World Health Organisation. 2020. Available at: https://www.who.int/news-room/detail/03-032020-shortage-of-personal-protective-equipment-endangeringhealth-workers-worldwide. 
[20] Zhang M, Zhou M, Tang F, Wang Y, Nie H, Zhang L, You G. Knowledge, attitude, and practice regarding COVID-19 among healthcare workers in Henan. Journal of Hospital Infection. 2020; 105: 183-187. Available at: https://www.journalofhospitalinfection.com/article/S01956701(20) 30187-0/fulltext).

[21] Chou R, Dana T, Buckley D, Selph S. Epidemiology of and risk factors for Coronavirus infection in Health Care Workers. Annals of internal medicine. 2020. Available at: https://www.acpjournals.org/doi/10.7326/M20-1632.

[22] Li R. C, Chung D. L. The role of hospital infection control in flattening the covid-19 curve: lessons from south korea. Health affairs. 2020. Available at: https://www.healthaffairs.org/do/10.1377/hblog20200511.415 767/full/.

[23] Wee LE, Conceicao EP, Sim XYJ, et al. Minimizing intrahospital transmission of COVID-19: the role of social distancing. J Hosp Infect. 2020; 105 (2): 113-115. doi: 10.1016/j.jhin.2020.04.016.

[24] Olum R, Chekwech G, Wekha G, Nassozi R and Bongomin F. Coronavirus Disease-2019: Knowledge, Attitude, and Practices of Health Care Workers at Makerere University Teaching Hospitals, Uganda. Frontier Public Health. 2020 https://doi.org/10.3389/fpubh.2020.00181.

[25] Khamlub S, Harun-Or-Rashid M, Sarker MA, Hirosawa T, Outavong P, Sakamoto J. Job satisfaction of health-care workers at health centers in Vientiane Capital and Bolikhamsai Province, Lao PDR. Nagoya J Med Sci. 2013; 75 (3-4): 233-241. Available at: https://www.ncbi.nlm.nih.gov/pmc/articles/PMC4345678/.

[26] Meng R, Li J, Zhang Y, et al. Evaluation of Patient and Medical Staff Satisfaction regarding Healthcare Services in Wuhan Public Hospitals. Int J Environ Res Public Health. 2018; 15 (4): 769. doi: 10.3390/ijerph15040769. 